\title{
Numerical simulation of the effects of diaphragm length on potential flow around a circular cylinder with rear diaphragm
}

\author{
Bofeng Fan ${ }^{1 *}$, Qingxiang Shui², Yuling Yang ${ }^{1}$ \\ ${ }^{1}$ School of Environment and Resources, Southwest University of Science and Technology, Mianyang 621010, China \\ ${ }^{2}$ School of Human Settlement and Civil Engineering, Xi' an Jiaotong University, Xi' an 710054, China
}

Corresponding Author Email: fbfyfbf@163.com

https://doi.org/10.18280/ijht.360232

Received: 1 October 2017

Accepted: 22 February 2018

\section{Keywords:}

finite-element analysis, rear diaphragm, potential flow around a circular cylinder, multi-step format (MSF), characteristicbased operator splitting $(C B O P)$

\begin{abstract}
To disclose the flow field features around a circular cylinder with rear diaphragm, this paper numerically simulates the effects of diaphragm length on the potential flow around such a circular cylinder, using the characteristic-based operator splitting (CBOP) method based on multi-step format (MSF). Through the simulation, the author obtained the flow field velocities, mean drag coefficient, lift coefficient and Strouhal number, and analysed the variation laws of the flow field features. The research shows that the addition of the rear transverse diaphragm can effectively suppress the vortex shedding in the wake region, reduce the pressure difference between the upper and lower surfaces of the circular cylinder, and greatly improve the flow around the circular cylinder. When the diagram length was sufficiently long, the vorticity of the upper and lower shear layers was completely dissipated during the backward movement along the transverse diaphragm, eliminating the occurrence of vortex shedding. The simulated laws of the flow velocities and flow field eigenvalues were consistent with the results of the previous studies. The research findings provide a valuable reference for similar studies in future.
\end{abstract}

\section{INTRODUCTION}

In computational fluid dynamics, the potential flow around a circular cylinder is a classical problem in various engineering applications, such as engineering and civil engineering. When the fluid bypasses a circular cylinder, boundary separation occurs on the surface of the structure, and flow separation and vortex shedding behind the cylinder at a certain Reynolds number. The long-time interaction between the unsteady separation flow and its accompanying vortex will induce structural fatigue and even resonance to the structure, exerting a negative impact on structural reliability and safety [1]. To reduce the undesirable impact, it is necessary to clarify the interaction mechanism between the fluid and the circular cylinder and identify the features of flow separation and vortex shedding in the influencing scope.

Much attention has been paid to the unsteady separation flow around a circular cylinder and the accompanying vortex movements. Reference [2] shows the existence of flow separation, free shear layer transition and other complex phenomena in the wake region of the circular cylinder. The vortex formation and shedding are the results of a complex mechanism. Reference [3] suggests that free shear layer transition is closely related to such parameters as flow free turbulence, boundary conditions of the end wall and lengthdiameter ratio of the circular cylinder. Focusing on 2D bluff bodies, Reference [4] attributes vortex formation and shedding to the entrainment and interaction between the upper and lower shear layers. Following this train of thoughts, Reference [5] adds a transverse diaphragm behind the circular cylinder, explores the effects of the relative position and length of the diaphragm on vortex formation and shedding, and puts forward a method to limit vortex shedding and alter the lift resistance coefficient.

Considering the complex interaction between the upper and lower shear layers of the potential flow around a circular cylinder, Reference [6] arranges wedge diaphragms along symmetry lines in the flow direction at the back of the circular cylinder and connects them with the cylinder; then, the 2D Navier-Stokes (NS) equations were solved by finite-element method, unstructured grid, and the laminar flow model, and applied to simulate the potential flow around the said circular cylinder [6]. The solution of the N-S equations is the key to the numerical simulation of the potential flow around a circular cylinder. Finite-element methods, such as Galerkin method and other new approaches [7-8], are desirable tools to solve flow problems with complex geometric boundaries.

If the Reynolds number is relatively large, however, the convection becomes the dominant flow pattern, resulting in an obvious nonlinearity of the N-S equations. In this case, the numerical solution acquired by the Galerkin method may oscillate violently. To solve the problem, Wang Daguo et al. [9] combined the characteristic-based split (CBS) method and the operator splitting method into the characteristic-based operator splitting (CBOS) method for the solution of N-S equations. This finite-element method is easy to implement with a small computing load per time step and easy to implement. However, the calculation efficiency of this method is rather low, because the calculation is only stable under small time steps. Shui Qingxiang et al. [10-11] created characteristic-based operator splitting method considering the multi-step format (CBOS-MSF), which can achieve high computing accuracy at any time step. This method was applied to simulate the potential flow around a circular cylinder with a 
rear diaphragm at different diaphragm lengths. The results show that this method can accurately simulate the small-scale physical quantities in the flow and suppress the pressure oscillation.

In light of the above, this paper adopts the CBOS-MSF to further simulate the potential flow around a circular cylinder with rear diaphragm at different diagram lengths, aiming to clarify the interaction mechanism between the fluid and the circular cylinder.

\section{CALCULATION MODEL}

The structure and computational grids of a circular cylinder with a rear diaphragm are illustrated in Figure 1, where $L$ is the length of the transverse diaphragm, $R$ is the radius of the circular cylinder, and $S$ is the thickness of the transverse diaphragm $(S=R / 25)$. During the simulation, only the value of $L$ was changed to disclose the effects of different diaphragm lengths on the potential flow around the circular cylinder. As shown in Figure 2, the flow calculation domain is 30D in the direction of the main flow, including 10D upstream the circular cylinder and $18 \mathrm{D}$ in the lateral dimension. The circular cylinder diameter $\mathrm{D}=1 \mathrm{~m}$ is the characteristic length

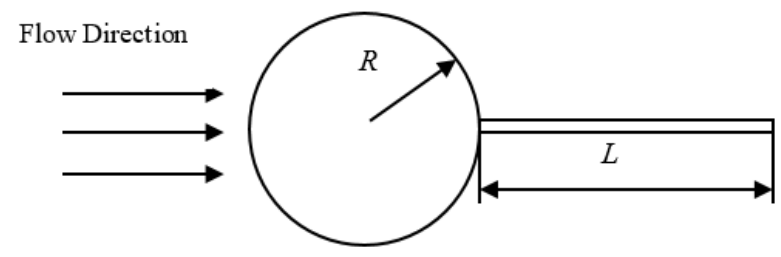

Figure 1. Structure of a circular cylinder with a rear diaphragm

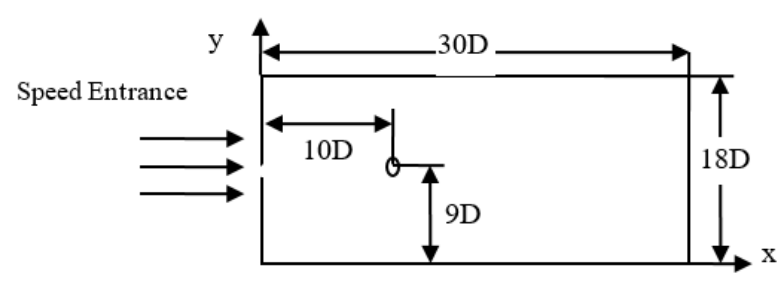

Figure 2. Calculation domain

\section{NUMERICAL MODEL}

\subsection{Governing equation}

The dimensionless form of the basic governing equation for a 2D unsteady incompressible fluid can be expressed as:

$$
\begin{gathered}
\left\{\begin{array}{c}
\frac{\partial u_{i}}{\partial t}+u_{j} \frac{\partial u_{i}}{\partial x_{j}}=f_{i}-\frac{\partial p}{\partial x_{i}}+\frac{1}{\operatorname{Re}} \frac{\partial}{\partial x_{j}}\left(\frac{\partial u_{i}}{\partial x_{j}}+\frac{\partial u_{j}}{\partial x_{i}}\right) \\
\frac{\partial u_{i}}{\partial x_{i}}=0
\end{array}\right. \\
\Omega \times[0, T]
\end{gathered}
$$

where $\Omega$ and $[0, T]$ are respectively the space domain and the time domain; $\mathrm{i}, \mathrm{j}=1,2 ;\left(u_{i}, u_{j}\right)=(u, v)$, with $u$ and $v$ being the horizontal speed and the vertical speed, respectively; $p$ is the pressure; $t$ is the time; $R e=\rho U L / \mu$ is the Reynolds number, with $\mu, \rho, \mathrm{U}$ and $\mathrm{L}$ being the dynamic viscosity, fluid density, characteristic speed and characteristic length, respectively; $f_{i}$ is the external force with $\left(x_{1}, x_{2}\right)=(x, y)$

\subsection{CBOS finite-element method}

In each time step, the N-S equations can be divided into a diffusion term and a convection term below by the operator splitting method:

$$
\begin{aligned}
& \left\{\begin{array}{l}
\frac{\partial u_{i}^{n+\theta}}{\partial t}-\frac{1}{\operatorname{Re}} \frac{\partial}{\partial x_{j}}\left(\frac{\partial u_{i}^{n+\theta}}{\partial x_{j}}+\frac{\partial u_{j}^{n+\theta}}{\partial x_{i}}\right)=-\frac{\partial p^{n+1}}{\partial x_{i}}+f_{i} \\
\frac{\partial u_{i}^{n+\theta}}{\partial x_{i}}=0
\end{array}\right. \\
& \frac{\partial u_{i}^{n+1}}{\partial t}+u_{j}^{n+1} \frac{\partial u_{i}^{n+1}}{\partial x_{j}}=0
\end{aligned}
$$

where $u_{i}^{n+\theta}$ is the solution of the diffusion term (2) at moment $n+1$ and the initial value of the convection term (3) at moment $n+1 ; u_{i}^{n+1}$ is the solution of the convection term at moment $n+1$, and the solution of the N-S equations (1) at moment $n+1$.

Using the CBOS finite-element method [9], equations (2) and (3) can be expressed in the following time-discrete forms:

$$
\begin{aligned}
& \left\{\begin{array}{l}
\frac{u_{i}^{n+\theta}-u_{i}^{n}}{\Delta t}-\frac{1}{\operatorname{Re}} \frac{\partial}{\partial x_{j}}\left(\frac{\partial u_{i}^{n+\theta}}{\partial x_{j}}+\frac{\partial u_{j}^{n+\theta}}{\partial x_{i}}\right)=-\frac{\partial p^{n+1}}{\partial x_{i}}+f_{i} \\
\frac{\partial u_{i}^{n+\theta}}{\partial x_{i}}=0
\end{array}\right. \\
& u_{i}^{n+1}-u_{i}^{n+\theta}=-\Delta t u_{j}^{n+\theta} \frac{\partial u_{i}^{n+\theta}}{\partial x_{j}} \\
& +\frac{\Delta t^{2} u_{k}^{n+\theta}}{2} \frac{\partial}{\partial x_{k}}\left(u_{j}^{n+\theta} \frac{\partial u_{i}^{n+\theta}}{\partial x_{j}}\right)
\end{aligned}
$$

\subsection{CBOS-MSF finite-element method}

The MSF was introduced to solve the convection term (3): splitting each time step $\Delta t$ into $h$ sub-steps and solving the convection term in each sub-step. Thus, the time step for solving the convection term can be defined as $\delta_{t}=\Delta t / h$. According to equation (5), the convection term in the $l$-th substep can be solved by the following formula:

$$
\begin{gathered}
u_{i}^{n+\frac{l}{h}}-u_{i}^{n+\frac{l-1}{h}}=-\delta t u_{j}^{n+\frac{l-1}{h}} \frac{\partial u_{i}^{n+\frac{l-1}{h}}}{\partial x_{j}} \\
+\frac{\delta t^{2}}{2} u_{k}^{n+\frac{l-1}{h}} \frac{\partial}{\partial x_{k}}\left(u_{j}^{n+\frac{l-1}{h}} \frac{\partial u_{i}^{n+\frac{l-1}{h}}}{\partial x_{j}}\right)
\end{gathered}
$$


where $l$ belongs to the interval $[1, h]$; If $l=1$, then $u_{i}^{n+\frac{l-1}{h}}=$ $u_{i}^{n+\theta}$; if $l=h$, then $u_{i}^{n+\frac{l}{h}}=u_{i}^{n+1}$.

The Galerkin method of weighted residuals was employed for the spatial discretization of equations (4) and (6). The solution process of the CBOS-MSF finite-element method is as follows:

(1) Obtain the transition value of the speed field $u_{i}^{n+\theta}$ and the pressure field $p^{n+1}$ at moment $n+1$ by solving equation (2) with the speed field $u_{i}^{n}$ and pressure field $p^{n}$ at moment $n$ as the initial values.

(2) Obtain the speed field $u_{i}^{n+1}$ according to equation (3) with $u_{i}^{n+\theta}$ as the initial value. During the solution of equation (3), the multi-step explicit processing (Figure 1) should be adopted.

(3) Go to the next moment and repeat steps (1) and (2).

The calculation process is shown in Figure 3.

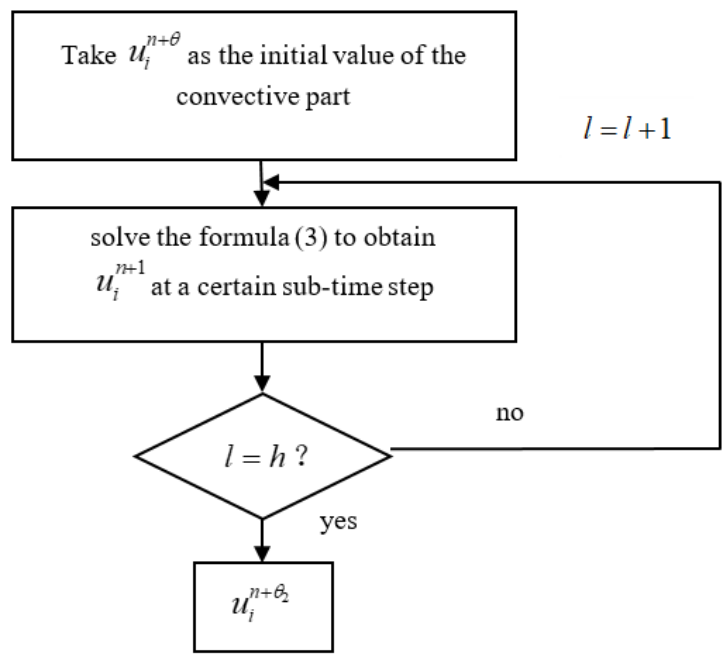

Figure 3. The solution process of the CBOS-MSF finiteelement method

\subsection{Calculation domain and boundary conditions}

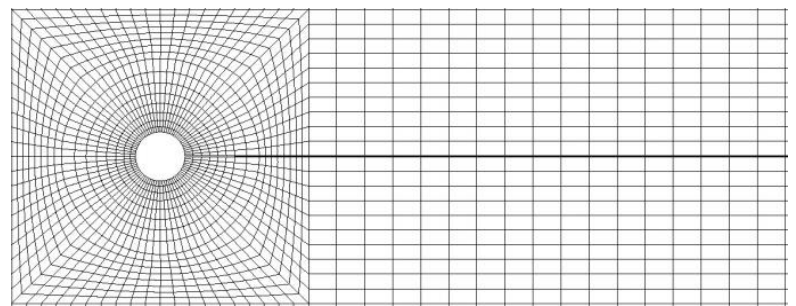

Figure 4. The calculation grids for the potential flow around the circular cylinder

The entire calculation domain was meshed into 11,860 fournode quadrilateral elements. In total, there were 12,114 nodes and 128 grids on the surface of the circular cylinder. The calculation grids are shown in Figure 4. The sidewall and surface were simulated as solid boundaries, the diaphragm surfaces as non-slip boundaries, the outlet as free flow boundaries (relative pressure: 0). It is assumed that the incoming flow is uniform with the horizontal speed $u=1$ and vertical speed $v=0$, and the initial values obey $u=v=0$. The other parameters are as follows: $U=1 \mathrm{~m} / \mathrm{s}, \rho=1.0 \mathrm{~kg} / \mathrm{m}^{3}$, substep of convection flow $h=10$, time step $=0.1 \mathrm{~s}$, and Reynolds number $R e=200$. In addition, the diaphragm length was set to different lengths during the simulation.

\section{SIMULATION RESULTS AND ANALYSIS}

\subsection{Simulation results}

The above model and algorithm were implemented to simulate the potential flow around the circular cylinder with a rear diaphragm under $R e=200$ and a uniform incoming flow. Figure 5 presents the flow velocities around the circular cylinder at different diaphragm lengths; Table 1 compares the mean drag coefficient $\overline{C_{D}}$, lift coefficient $C_{L}$ and Strouhal number $S_{t}$ calculated in this paper with those presented in relevant references $[12,13]$; Table 2 lists the mean drag coefficient $\overline{C_{D}}$, lift coefficient $C_{L}$ and Strouhal number $S_{t}$ of each diaphragm length; Figures 6 and 7 show the variations of Strouhal number $S_{t}$ and the mean drag coefficient $\overline{C_{D}}$ with diaphragm lengths.
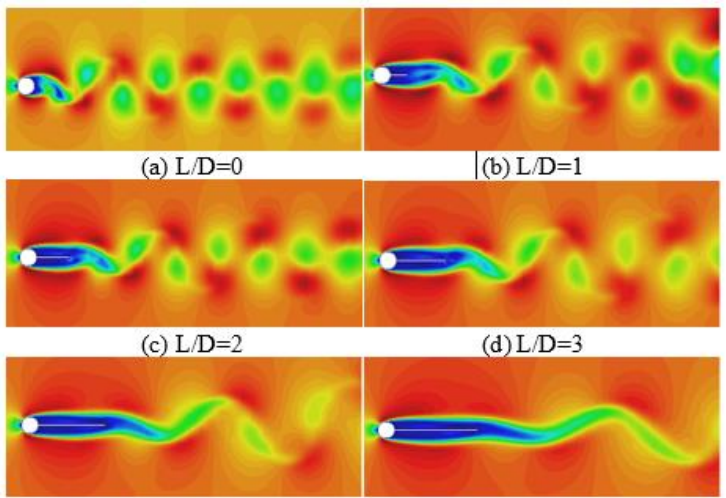

(e) $\mathrm{L} / \mathrm{D}=4$

(f) $\mathrm{L} / \mathrm{D}=5$

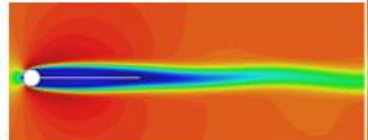

(g) $\mathrm{L} / \mathrm{D}=6$

Figure 5. Flow velocities around the circular cylinder at different diaphragm lengths $(R e=200)$

Table 1. Comparison between the calculated results with the data of other references $(R e=200)$

\begin{tabular}{cccc}
\hline Calculation results & $\overline{C_{D}}$ & $C_{L}$ & $S_{t}$ \\
\hline This paper & 1.339 & \pm 0.614 & 0.194 \\
Ding et a ${ }^{[12]}$ & 1.348 & \pm 0.659 & 0.196 \\
Harichandan et al $^{[13]}$ & 1.33 & \pm 0.60 & 0.192 \\
\hline
\end{tabular}

Table 2. Flow coefficient values around the circular cylinder at different diaphragm lengths $(R e=200)$

\begin{tabular}{ccccccccccccccccccc}
\hline $\mathrm{L} / \mathrm{D}$ & 0 & 0.5 & 1 & 1.5 & 2 & 2.5 & 3 & 3.5 & 4 & 4.5 & 5 & 5.5 & 6 & 6.5 & 7 & 0.945 \\
\hline $\bar{C}_{D}$ & 1.339 & 1.165 & 1.0624 & 1.062 & 1.0614 & 1.071 & 1.0228 & 1.0198 & 0.9748 & 0.9616 & 0.9589 & 0.9521 & 0.9512 & 0.9439 & 0.9435 \\
$C_{L}$ & \pm 0.614 & \pm 0.333 & \pm 0.163 & \pm 0.157 & \pm 0.154 & \pm 0.169 & \pm 0.179 & \pm 171 & \pm 0.155 & \pm 0.139 & \pm 0.113 & \pm 0.0864 & \pm 0.064 & \pm 0.0561 & 0 & \\
$S_{t}$ & 0.194 & 0.167 & 0.155 & 0.164 & 0.178 & 0.172 & 0.154 & 0.138 & 0.123 & 0.111 & 0.0984 & 0.08961 & 0.0808 & 0.0745 & 0 & \\
\hline
\end{tabular}




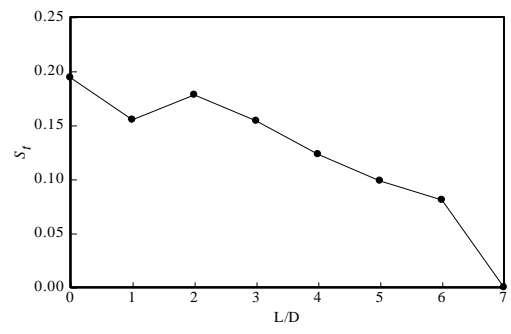

Figure 6. The variation curve of $S_{t}$ at different diaphragm lengths $(R e=200)$

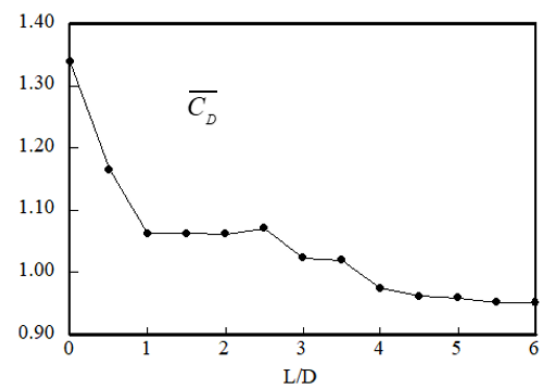

Figure 7 The variation curve of $\overline{C_{D}}$ at different diaphragm lengths $(R e=200)$

\subsection{Results analysis}

As shown in Table 1 , the $\overline{C_{D}}, C_{L}$ and $S_{t}$ calculated in this paper agree well with the data in the references, indicating that our algorithm can accurately simulate the flow field features around the circular cylinder. According to the flow velocities in Figure 5, when there was no diaphragm, vortexes were produced through the interaction between the upper and lower free shear layers, creating a wake region behind the circular cylinder; in this region, the vortexes appeared and disappeared alternately, and formed a stable vortex street. After the addition of the diaphragm, the flow around the circular cylinder underwent significant changes: the shear layers were confined to the sides downstream the diaphragm, exhibiting an attenuating trend; it was not until the end of the diaphragm that a vortex was generated through the interaction between the shear layers. With the increase of the diaphragm length, the starting point of vortex shedding moved back in the flow direction. At the diaphragm length of $\mathrm{L} / \mathrm{D}=7$, the transverse diaphragm completed blocked the interaction between the shear layers on the sides of the circular cylinder. In this case, the vorticity of the upper and lower shear layers was completely dissipated during the backward movement along the transverse diaphragm, eliminating the occurrence of vortex shedding.

Figure 6 shows the variation curve of the Strouhal number $S_{t}$ at different diaphragm lengths $(R e=200)$. With the increase of the diaphragm length, the $S_{t}$ decreased when $0<L / D<1$, increased when $1<L / D<2$, and decreased again when $2<L / D$; when $L / D=7, S_{t} \approx 0$, indicating the disappearance of vortex shedding. These trends are consistent with Figure 5(h), where no vortex shedding exists, and the results of Reference [6].

Figure 7 shows the variation curve of the mean drag coefficient $\overline{C_{D}}$ at different diaphragm lengths $(R e=200)$. It can be seen that the mean drag coefficient $\overline{C_{D}}$ decreased with the increase of the diaphragm length. From the $C_{L}$ value in Table 2 , it is learned that the addition of the transverse diaphragm significantly reduced the pressure coefficient difference between the upper and lower surfaces of the circular cylinder. Due to its consistency with the difference, the amplitude of lift coefficient on the circular cylinder also declined dramatically. In addition, the amplitude of lift coefficient was reduced, but not to zero, when the transverse diaphragm length was equal to the diameter of the circular cylinder. Under the same conditions, this amplitude dropped to zero in Reference [14].

To explain why the amplitude of the lift force was reduced, but not to zero, when the transverse diaphragm length was equal to the diameter of the circular cylinder, the pressure coefficient $C_{p}$ distribution on the surfacse of the circular cylinder was investigated when the lift coefficient reached the maximum at the positive direction at $R e=200$. The distribution is shown in Figure 8, where the solid line is the distribution without transverse diaphragm and the dashed line is the distribution with transverse diaphragm. According to the figure, when the positive lift coefficient reached the maximum, the pressure coefficients of the upper layer $\left(90^{\circ}<\theta<180^{\circ}\right)$ and lower layer $\left(180^{\circ}<\theta<270^{\circ}\right)$ behind the circular cylinder were increased after the addition of the transverse diaphragm. Comparatively, the upper layer had a greater pressure increment than the lower layer. Therefore, the transverse diaphragm reduced but not eliminated the pressure coefficient difference between the upper and lower surfaces of the circular cylinder. Due to its consistency with the difference, the amplitude of lift coefficient on the circular cylinder with transverse diaphragm also declined dramatically, but not to zero.

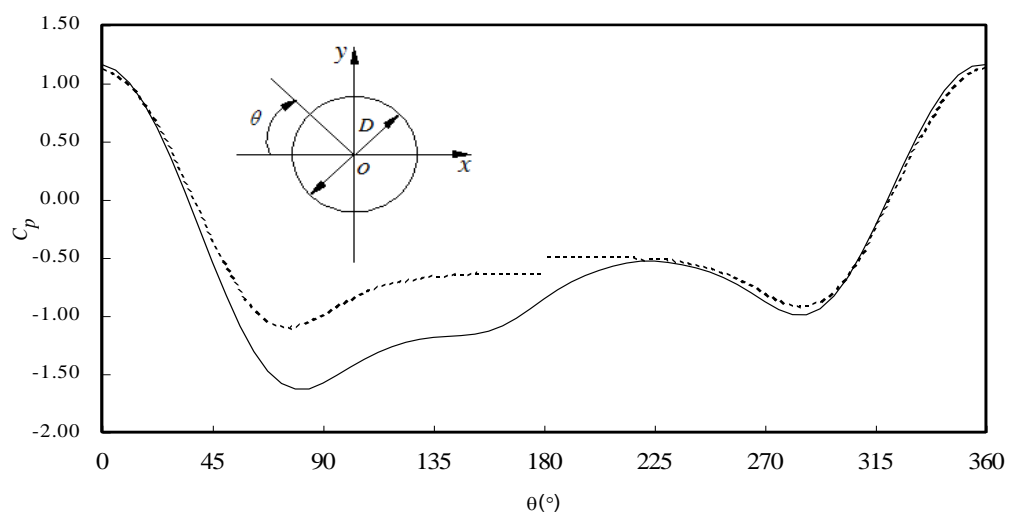

(Solid line: the distribution without transverse diaphragm; dashed line: the distribution with transverse diaphragm)

Figure 8. Pressure coefficient distribution on the surfaces of the circular cylinder when the lift coefficient reached the maximum at the positive direction $(R e=200)$ 


\section{CONCLUSIONS}

In this paper, the CBOS-MSF finite-element method was adopted to simulate the potential flow field around a circular cylinder with rear transverse diaphragm at different diaphragm lengths under $R e=200$. The simulation shows that the addition of the rear transverse diaphragm can effectively suppress the vortex shedding in the wake region, reduce the pressure difference between the upper and lower surfaces of the circular cylinder, and greatly improve the flow around the circular cylinder. When the diagram length was sufficiently long $(L / D=7)$, the vorticity of the upper and lower shear layers was completely dissipated during the backward movement along the transverse diaphragm, eliminating the occurrence of vortex shedding. The simulated laws of the flow velocities and flow field eigenvalues were consistent with the results of the previous studies. The research findings provide a valuable reference for similar studies in future.

\section{REFERENCE}

[1] Li QH, Liu GM, Xue K, Wang J, Wang P. (2015). Free vibration analysis of cylindrical orthotropic circular plates. Journal of Harbin Engineering University 36(7): 981-986. https://doi.org/10.3969/j.issn.10067043.201404082

[2] Ye CM, Wu WQ. (1997). Numerical simulation of vortex movement for the flow past a circular cylinder and the analysis of wake instability. Journal of Engineering Thermophysics 18(2): 169-172.

[3] Ling GC, Yin XY. (1982). Secondary vortex and the process of the formation of karman vortex. Chinese Journal of Theoretical and Applied Mechanics 18(1): 18$25,111-112$.

[4] Gerrard JH. (1966). The mechanics of the formation region of vortices behind bluff bodies. Journal of Fluid Mechanics 25(2): 401-413. https://doi.org/10.1017/S0022112066001721

[5] Yoon J, Kim J, Choi H. (1996). Control of laminar vortex shedding behind a circular cylinder using tabs. Physics of Fluids, 8(2): 479-486. https://doi.org/10.1007/s12206014-0317-x

[6] He C, Duan ZQ. (2012). Numerical simulation of twodimensional laminar flow around circular cylinder with splitter plate. Journal of Southwest Jiaotong University 47(5): 826-830. https://doi.org/10.3969/j.issn.02582724.2012.05.015

[7] Hughes TJR, Franca P, Hulbert GM. (1989). A new finite element formulation for computational fluid dynamics: VIII. The galerkin/least-squares method for advective diffusive equations. Computer Methods in Applied Mechanics and Engineering 73(2): 173-189. https://doi.org/10.1016/0045-7825(89)90111-4

[8] Tan L, Zhu BS, Wang YC, et al. (2014). Turbulent flow simulation using large eddy simulation combined with characteristic-based split scheme. Computers\& Fluids 94(1): 161-172. https://doi.org/10.1016/j.compfluid.2014.01.037

[9] Wang DG, Wang HJ, Xiong JH, Tham LG. (2011). Characteristic-based operator-splitting finite element method for navier-stokes equations. Science China Technological Sciences 54(8): 2157-2166. https://doi.org/10.1007/s11431-011-4444-7

[10] Glowinski R, Pironneau O. (1992). Finite element method for the Navier-Stokes equations. Annual Review of Fluid Mechanics 24(1): 167-204.

[11] Shui QX, Wang DG, Wang W. (2016). Numerical Simulation of the characteristics of flow past circular cylinder with splitter plate. Advances in Water Science 27(4): $586-592$. https://doi.org/10.14042/j.cnki.32.1309.2016.04.013

[12] Ding H, Shu C, Yeo K S, Xu D. (2007). Numerical simulation of flows around two circular cylinders by mesh-free least square-based finite difference methods. International Journal for Numerical Methods in Fluids 53(2): 305-332. https://doi.org/10.1002/fld.1281

[13] Harichandan AB, Roy A. (2010). Numerical investigation of low Reynolds number flow past two and three circular cylinders using unstructured grid CFR scheme. International Journal of Heat and Fluid Flow 31(2): 154171. https://doi.org/10.1016/j.ijheatfluidflow.2010.01.007

[14] He C, Duan ZQ. (2012). Numerical simulation of twodimensional laminar flow around circular cylinder with splitter plate. Journal of Southwest Jiaotong University 47(5): 826-831. https://doi.org/10.3969/j.issn.02582724.2012.05.015 